\title{
AR $(p)$ PARA PREVISÃO DE DEMANDA EM UMA AGROINDÚSTRIA ${ }^{1}$
}

\author{
Álvaro de Aquino Santos² \\ Marcos Antônio Alves \\ Elias Silva de Medeiros \\ Igor Neves Nunes \\ Rafael João de Melo Miguel Cardoso
}

\begin{abstract}
RESUMO
Os modelos de previsão de séries temporais são ferramentas importantes no apoio ao planejamento da produção e na tomada de decisão de organizações como as agroindústrias. Um dos desafios da agroindústria brasileira de frango, uma das mais importantes do mundo, é prever adequadamente a demanda de seus clientes. Nesse contexto, o objetivo deste estudo foi propor um modelo paramétrico para previsão de demanda baseado nos dados de expedição de pintos da linhagem de corte em uma agroindústria do centro-oeste de Minas Gerais. Diferentes métodos foram avaliados sobre a série histórica de 59 semanas, a fim de identificar o comportamento das expedições semanais e verificar possíveis tendências e sazonalidades. Dados de 56 semanas foram avaliados e os modelos candidatos foram obtidos a partir da análise das autocorrelações das observações. Por meio dos critérios de avaliação BIC e AIC, o modelo Autorregressivo de Primeira Ordem (AR) (1) se mostrou o mais adequado. Para avaliar o poder de predição do modelo AR (1) foi realizada uma comparação entre os valores preditos e observados nas últimas quatro semanas da série. Por meio da análise foi verificado um desempenho satisfatório, uma vez que os valores observados se encontravam dentro do intervalo de $95 \%$ de confiança construído por meio do modelo.
\end{abstract}

Palavras-chave: Série temporal. Previsão de demanda. Pintos de corte. Frango.

\section{AR $(p)$ FOR FORECASTING DEMANDDATA IN AN AGROINDUSTRY}

\begin{abstract}
Time series forecasting models are important tools in supporting production planning and decision-making of the companies, such as agroindustries. One of the challenges facing Brazilian chicken agribusinesses, one of the most important in the world, is to correctly forecast the demand of its customers. In this context, the aim of this paper was to propose a parametric model for demand forecasting based on the data of dispatch of chicks of the broiler line in an agroindustry in the Midwest of Minas Gerais. Different methods were evaluated over the 59-week historical series in order to identify the behavior of the weekly expedition and to verify possible trends and seasonalities. Data of 56 weeks were evaluated and the candidate models were obtained from the analysis of the autocorrelations of the observations. Through the BIC and AIC evaluation criteria, the First Order Autoregressive model (AR) was the most appropriate one.
\end{abstract}

\footnotetext{
${ }^{1}$ Como citar este artigo: SANTOS, Á. A.; ALVES, M. A.; MEDEIROS, E. S.; NUNES, I. N.; CARDOSO, R. J. M. M. AR (p) para previsão de demanda em uma agroindústria. ForScience, Formiga, v. 8, n. 1, e00507, jan./jun. 2020. DOI: 10.29069/forscience.2020v8n1.e507.
}

${ }^{2}$ Autor para correspondência: Álvaro de Aquino Santos, e-mail: aasalvarosamonte@gmail.com. 
To assess the predictive accuracy of the AR (1) model, a comparison was made between predicted and observed values in the last four weeks of the time-series. Through this analysis, a satisfactory performance was verified, since the observed values were within the $95 \%$ confidence interval constructed through the model.

Keywords: Time-series. Demand forecasting. Broiler chicks. Chicken.

\section{INTRODUÇÃO}

No atual mundo dos negócios, as empresas estão sujeitas a diversos fatores que ameaçam a sua sobrevivência. As flutuações do mercado de capitais, suprimentos, mão-de-obra e previsão de demanda são alguns impulsionadores de um ambiente competitivo e instável (LEMOS, 2016). Nesse contexto, um dos pontos importantes para a manutenção de uma empresa, como as agroindústrias, é a capacidade de planejamento operacional (MARTINS; LAUGENI, 2005; CHIAVENATO, 2008; SIMCHI-LEVI; KAMINSKY; SIMCHI-LEVI, 2010).

As agroindústrias de frango de corte brasileiras estão entre as maiores e mais importantes do mundo (ABPA, 2019; PAIVA; HERVAL; SÁFADI, 2019). Elas precisam continuadamente ajustar sua demanda exatamente de acordo com as necessidades dos clientes, sejam nacionais $(68 \%)$ ou estrangeiros (32\%) (ABPA, 2019). Adiciona-se àquelas que lidam com pintos da linhagem de corte um fator agravante: seus produtos são carga viva e devem ser comercializados no dia do nascimento, pois não há locais para armazenamento e perdem o valor comercial.

Nesse âmbito, uma maneira de mensurar essa demanda de expedição é por meio de modelos de séries temporais, sejam paramétricos ou não-paramétricos. Eles podem ser utilizados para modelar dados nas áreas de economia e finanças (ALVAREZ-RAMIREZ; RODRIGUEZ, 2018; MORETTIN; TOLOI, 2018). Como aplicações práticas, incluem-se: previsão da oferta de hortaliças para uma rede de supermercados (BRANCO et al., 2018), demanda de ração de peixe (UEDA et al., 2018), demanda de produtos naturais (SANTOS et al., 2019) e abate de frangos de corte (PAIVA; HERVAL; SÁFADI, 2019). Em relação à previsão de produção de carne de frango, Paiva, Herval e Sáfadi (2019) propuseram um modelo paramétrico para analisar a série de abate de frangos no Brasil utilizando dados de 2000 a 2018. O modelo autorregressivo integrado de médias móveis sazonal (SARIMA) foi o escolhido. Os resultados indicaram uma queda para o ano de 2019, fato este corroborado pelo relatório da Associação Brasileira de Proteína Animal (ABPA, 2019). Quanto à comercialização da carne, a ABPA dá apontamentos de pesquisas que vêm sendo desenvolvidas sobre o melhoramento genético e na alimentação dos animais, além do elevado consumo nacional e as taxas de exportação. 
Embora essas pesquisas tragam importantes contribuições para a agroindústria de frango, o foco delas não está na previsão de expedição de pintos da linhagem de corte. Conforme antes indicado, as pesquisas são voltadas ou para a produção do frango de corte ou para aspectos de melhoramento genético ou composição alimentar. É sabido que realizar a previsão de demanda de um produto é uma tarefa complexa por muitos fatores, tais como: incertezas sobre o cenário econômico, confiabilidade e disponibilidade dos dados para pesquisa, dificuldade em selecionar e interpretar o método para previsão que seja condizente com as características do produto, da demanda real do mercado e do período a ser estimado. Além disso, a análise de dados históricos pode apresentar comportamentos bastante diferenciados de demanda por diferentes tipos de produtos, podendo ser suscetível a tendências ou sazonalidades (MORETTIN; TOLOI, 2018; SANTOS et al., 2019). Por isso, faz-se necessário a avaliação e escolha de um método mais condizente com as singularidades da série investigada.

Diante do exposto, esta pesquisa teve como objetivo geral propor um modelo paramétrico de previsão de expedição de pintos da linhagem de corte de uma agroindústria de grande porte localizada no centro-oeste mineiro. Os dados foram cedidos pela empresa e compreendem um período de 59 semanas (16/05/2016 a 02/07/2017). Como objetivos específicos, o estudo visou a) identificar o comportamento da série temporal a fim de identificar a existência ou não de tendência e sazonalidade, b) desenvolver e formular as previsões para validar a autorregressão como método de previsão de expedição e c) validar o modelo com resultados dentro de um intervalo de confiança, uma vez que se trata de um caso prático.

\section{REFERENCIAL TEÓRICO}

Os modelos de previsão de séries temporais são um forte apoio ao planejamento da operação dos projetos e na tomada de decisão (MARTINS; LAUGENI, 2005; CHIAVENATO, 2008; SIMCHI-LEVI; KAMINSKY; SIMCHI-LEVI, 2010). Dois pontos se destacam no contexto de previsão de demanda: o primeiro deles é que muitas empresas não exploram adequadamente os dados históricos para obter estimativas acuradas de demanda futura; e o segundo, conforme apontaram Simchi-Levi, Kaminsky e Simchi-Levi (2010), é que essas empresas projetam seus processos de planejamento como se a previsão feita muito antes da temporada de vendas fosse uma representação precisa da realidade. Contudo, uma ressalva feita por Tubino (2017) indica que a demanda necessita ser pensada em torno de prazo, seja curto, médio ou longo, dependendo do tipo de produto e produção. As previsões de longo prazo são utilizadas 
como base para elaboração do plano de produção, enquanto as de médio e curto prazo são empregadas para planejamento mestre e programação da produção.

$\mathrm{Na}$ agroindústria brasileira de carne de frango, umas das maiores e mais importantes do mundo (ABPA, 2019; PAIVA; HERVAL; SÁFADI, 2019), a pressão mercadológica por melhorias genéticas e pela demanda de produtos é ainda maior. De acordo com os dados do relatório da ABPA (2019), o consumo interno corresponde a 68\% da produção nacional, com consumo per capita médio de $40 \mathrm{~kg}$ por habitante (valor muito aproximado aos 10 anos anteriores) e $32 \%$ para exportação para outros continentes. Especificamente no ramo de pintos de corte, aqueles que são comercializados para recriação e posterior abate, é fundamental ter boas estimativas da quantidade de ovos a ser previamente encubada para atender aos clientes no momento adequado.

No que diz respeito ao melhoramento genético das aves, os dados apresentados pela ABPA (2019) indicam que vêm sendo realizadas pesquisas nesta área. A expectativa é de aumentar a produção e atender a demanda nacional e internacional, tanto nos elos da cadeia produtiva (SCHMIDT; SILVA, 2018), agroindústria de grande porte (THEOBALD et al., 2019), quanto pequenos produtores (RODRIGUES et al., 2011; DELABOUGLISE et al., 2019; OSINOWO; TOLORUNJU, 2019). Um dos casos é apresentado por Piqueti et al. (2018). Estes autores analisaram o aumento do ganho de peso diário dos frangos de corte de uma agroindústria catarinense e concluíram que era possível reduzir o tempo de criação dos frangos ao mesmo tempo possibilitava aumentar a produção anual.

Todavia, essas pesquisas antes mencionadas não objetivaram analisar os dados históricos da agroindústria e aplicar métodos de previsão de demanda. Esta previsão, segundo Tubino (2009), é a base para o planejamento estratégico, sendo a variável mais importante desenvolvida pelo planejamento e controle da produção. Assim, dada a grande importância da avicultura na economia nacional e seu impacto na produção mundial, a análise de séries temporais pode ser uma ferramenta útil no controle do sistema produtivo. Estes métodos podem ser tanto paramétricos, como os modelos autorregressivos e de médias móveis (MORETTIN; TOLOI, 2018; DE MEDEIROS et al., 2018) ou não-paramétricos, como os de séries temporais fuzzy (SILVA et al., 2017).

Recentemente, Paiva, Herval e Sáfadi (2019) propuseram uma análise de séries temporais para o abate de frango. Os autores utilizaram dados públicos de dezoito anos (2000-2018) e verificaram a aditividade do modelo, além de tendência ou sazonalidade. Os testes realizados e os baixos valores de erro padrão encontrados viabilizaram a aplicação do modelo SARIMA. 
Os resultados apontaram uma queda na quantidade de abate de frangos para o ano de 2019, o que é corroborado pelos dados apresentados no relatório da ABPA para o mesmo ano.

Há, no entanto, uma carência de pesquisas que busquem prever a expedição de pintos de corte pelas agroindústrias. A maior parte delas, incluindo o foco principal do relatório da ABPA, levam em consideração ou os frangos ou os ovos. A previsão de expedição de pintos de corte é um fator crítico para as agroindústrias por vários motivos. Dentre eles, dois se destacam: 1) não é interessante para as agroindústrias ter pintos com mais de um dia de vida, pois perdem o valor comercial; 2) a incubação dos ovos fertilizados deve ser feita três semanas antes do dia da venda, pois considera-se o tempo para os ovos chocarem. Há, ainda, outros fatores logísticos inseridos neste contexto, como o recebimento dos pedidos pelo cliente, separação e preparação dos ovos entre outros.

\section{MATERIAL E MÉTODOS}

\subsection{Material}

O conjunto de dados utilizado nesta pesquisa é referente a uma empresa de grande porte do setor de avicultura, localizada na região do centro-oeste de Minas Gerais. Por questões legais e de marca, manteve-se o anonimato da mesma. A empresa atua exclusivamente com o manejo de ovos para que possa ocorrer o nascimento de pintos de diferentes linhagens. Ela está consolidada há anos no mercado e possui outras unidades no território brasileiro.

Os pintos de corte são adquiridos pelos clientes principalmente para recriação. Posteriormente eles são abatidos e vendidos no varejo. O nascimento de um pinto de corte ocorre três semanas após a incubação. Além disso, a empresa precisa de uma semana para gerenciar seus processos internos, preparar transporte dos ovos e chocadeiras. Assim sendo, a previsão deve ser feita para quatro semanas antes do nascimento dos pintos.

A série histórica relacionada à expedição semanal de pintos de corte representa dados reais e foi obtida na empresa que busca melhorar seus processos internos. Foram escolhidos apenas os pintos da linhagem de corte, pois eles representam a maior parte da receita da empresa. A série é referente ao período de16/05/2016 a 02/07/2017.

\subsection{Métodos}


O modelo paramétrico autorregressivo (AR) relaciona a série temporal com seus valores históricos, visto que em o passado imediato é um bom ponto de partida para prever o futuro (STOCK; WATSON, 2012; OLIVEIRA; DOURADO; MELLO, 2017). Sendo assim, este trabalho pretende utilizar um modelo paramétrico de série temporal para previsão de demanda, para dar apoio ao sistema de produção da agroindústria. $\mathrm{O}$ interesse principal é obter uma série estacionária, e para isso é necessário obter uma série livre de tendência e sazonalidade. No caso de interesse em séries não-estacionárias e tendências, recomenda-se a leitura de Alves et al. (2018).

Em um modelo autorregressivo de médias móveis (ARMA), é assumido que o valor futuro de uma variável pode ser representado por uma função linear de várias observações passadas e de erros aleatórios (DE MEDEIROS et al., 2018). O modelo ARMA é expresso pela Equação (1)

$$
y_{t}=\theta_{0}+\sum_{i=1}^{p} \phi_{i} y_{t-1}-\sum_{j=1}^{q} \theta_{j} \varepsilon_{t-j}+\epsilon_{t}
$$

onde $y_{t}$ é a série observada no instante $t, t=1, \ldots, n, \epsilon_{t}$ é o erro aleatório associado à observação $y_{t}, \phi_{i}(i=1, \ldots, p)$ são os parâmetros autorregressivos e $\theta_{j}(j=1, \ldots, q)$ são os parâmetros de médias móveis e, por fim, $p$ e $q$ as ordens destes parâmetros. É fácil perceber que quando $q=0$ tem-se um modelo autorregressivo (AR) de ordem $p$. Quando $p=0$ o modelo passa a ser de média móvel (MA) de ordem $q$. Para este modelo admite-se que os erros aleatórios $\left(\epsilon_{t}\right)$ sejam ruído branco (RB), com média zero e variância constante $\sigma^{2}$, bem como não sejam autocorrelacionados $\left(\epsilon_{t} \sim R B\left(0, \sigma^{2}\right)\right.$.

Tanto o modelo AR quanto o modelo ARMA são formas de aproximar as autocovariâncias de $y_{t}$. Uma vez que a série temporal é identificada como estacionária, com variância finita, ela pode ser escrita com uma autorregressão ou com uma média móvel, desde que o termo de erro serial não seja correlacionado (BOX et al., 2015).

Para a escolha das ordens de um modelo autorregressivo pode-se considerar a autocorrelação encontrada nos dados, dado os valores anteriores. A função de autocorrelação (FAC) e função de autocorrelação parcial (FACP) são ferramentas usadas para evidenciar a correlação dos dados, em que podem ser gerados gráficos a fim de facilitar o processo (BUENO, 2008). A FAC define a defasagem do modelo MA. Já a FACP define a defasagem do modelo AR. No caso do $\operatorname{AR}(p)$, a FAC decai com o aumento das defasagens e a FACP é truncada a partir da 
defasagem $p$. Já no MA $(q)$ o inverso acontece, a FAC é truncada a partir da defasagem $q$ e a FACP decai. Em um ARMA $(p, q)$ a FAC decai a partir da defasagem $q$ e a FACP decai a partir da defasagem $p$ (BUENO, 2008). A Tabela 1 sumariza esta relação para melhor identificação dos modelos.

Tabela 1 - Identificação dos modelos

\begin{tabular}{ll}
\hline \multicolumn{1}{c}{ Modelo } & Identificação \\
AR $(p)$ & FAC decai e FACP é truncada a partir da defasagem $p$. \\
MA $(q)$ & FACP decai e FAC é truncada a partir da defasagem $q$. \\
ARMA $(p, q)$ & FAC decai a partir da defasagem $q$ e FACP decai a partir da defasagem $p$. \\
\hline
\end{tabular}
Fonte: Bueno (2008).

A identificação de um modelo através da FAC e FACP é um processo difícil. Por isso, é usual gerar todos os resultados possíveis e eleger aquele modelo cujos resíduos sejam RB e com o menor valor do critério de informação de Akaike (AIC) e Bayesiano (BIC) (BUENO, 2008; TANAKA, 2017; PAIVA; HERVAL, SÁFADI, 2019). Após os resultados, também é coeso calcular a raiz quadrada do erro quadrático médio (RMSE).

Neste trabalho, para a formulação dos cálculos, foi utilizado o software Gnu Regression, Econometricsand Time-series Library (Gretl). A escolha desse software se deve ao fato de ele ser livre, código aberto e muito utilizado no campo da econometria para previsão de séries temporais (GRETL, 2018).

Dadas estas informações, a seção a seguir apresenta a série temporal utilizada nesta pesquisa. Em seguida é realizado o estudo desta série, utilizando os modelos de previsão abordados, para identificar comportamentos e extrair informações importantes que os dados podem fornecer, a fim de escolher o melhor modelo e, consequentemente, obter os melhores resultados.

\section{RESULTADOS E DISCUSSÃO}

Para fins de análise de desempenho do modelo foram retiradas as quatro últimas semanas (visto a necessidade antes explicitada) com o intuito de verificar o erro gerado pela previsão quando comparado com os dados observados. Assim, para seleção do modelo foi utilizada a série temporal da expedição referente a 55 semanas (Figura 1). 


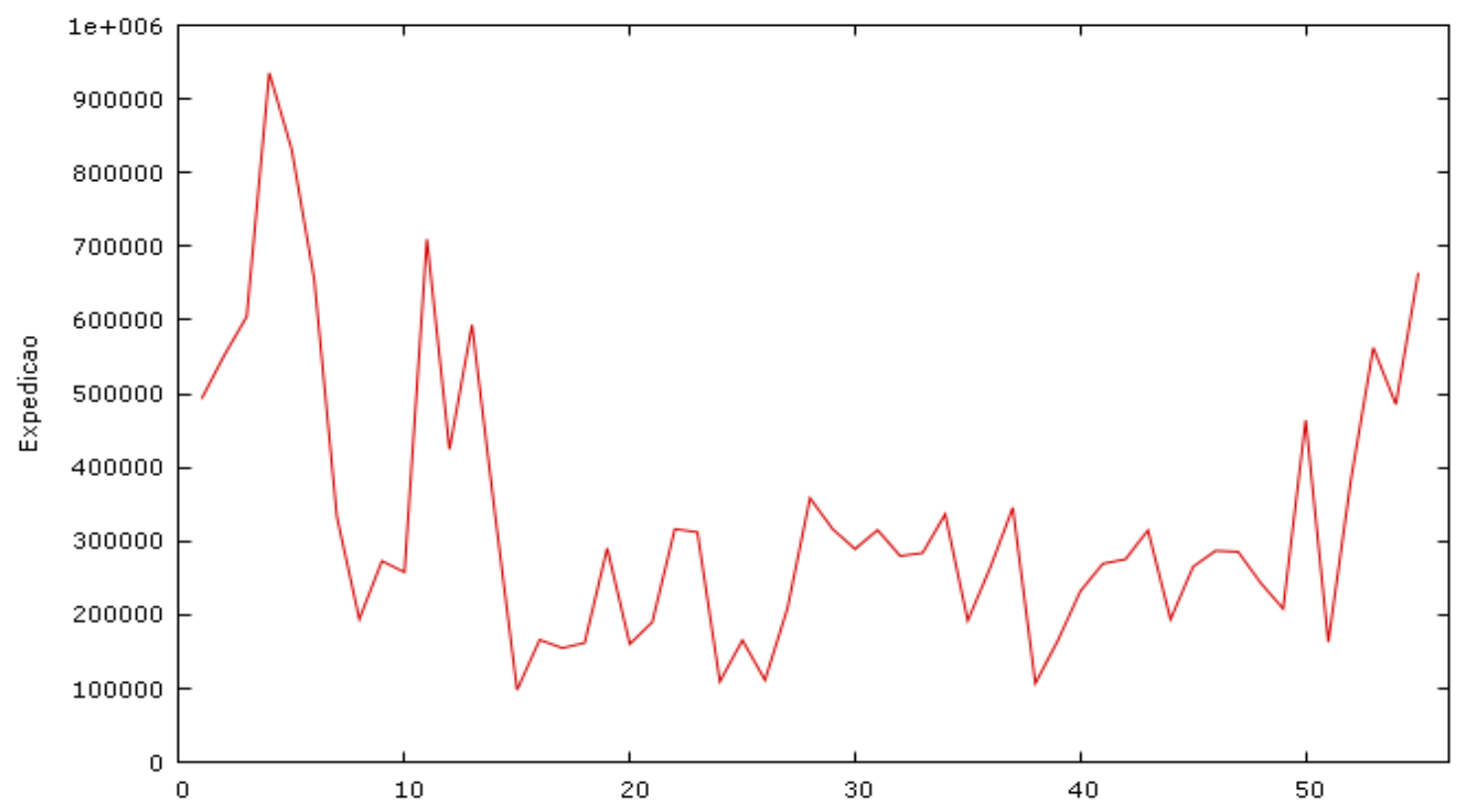

Figura 1 - Série temporal de expedição considerando as 55 semanas observadas Fonte: Autores (2018).

Para que possa ser vista a necessidade de transformação logarítmica $(\ln )$ dos dados verificou-se a relação amplitude versus média. Segundo De Medeiros et al. (2018), quando o resultado apresenta coeficiente de inclinação estatística significativo, tem-se a necessidade de uma transformação na série. O resultado apresentou um comportamento linear crescente entre a média e a amplitude sugerindo uma possível transformação na série. $O$ teste de hipótese para o coeficiente de inclinação deste ajuste foi feito e rejeitou-se a hipótese nula de que o coeficiente de inclinação é estatisticamente igual a zero com 0,01 de significância. Logaritmizar a série transforma o modelo multiplicativo em aditivo e estabiliza a variância.

Após a transformação foi necessário verificar o comportamento desta série temporal para identificar possíveis comportamentos de tendência e sazonalidade, conforme ilustrado na Figura 3a.É possível perceber visualmente que a série logaritmizada dos dados de expedição apresenta um comportamento constante. Logo, há indícios de que a série não possui tendência e, portanto, não se faz necessária a realização de uma diferença na parte estacionária. A fim de confirmar isso foi feito o teste de hipótese de DickeyFuller (PAPARODITIS; POLITIS, 2018). Após a aplicação deste teste, a hipótese nula de que a série possuía raiz unitária foi rejeitada a um nível de 0,01 de significância, uma vez que o valor p foi de 0,006 . Portanto não é necessária aplicar uma diferença nesta série, sugerindo desta forma que a série de expedição não possui tendência.

Quanto à sazonalidade, foi elaborado o perodograma (Figura 2b), bem como aplicado o teste de Kruskal-Wallis (KRUSKAL; WALLIS, 1952) sob a hipótese nula de não sazonalidade 
na série. Tanto o periodograma, quando o valor p obtido $(0,7678)$ indicam um comportamento não sazonal dos dados.

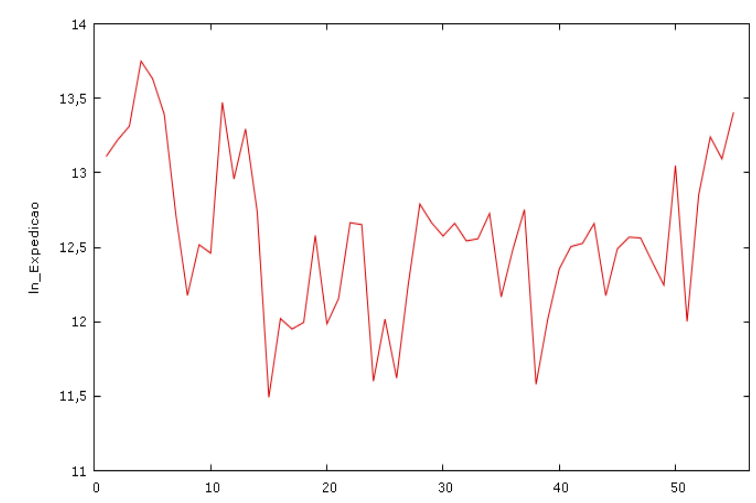

(a)

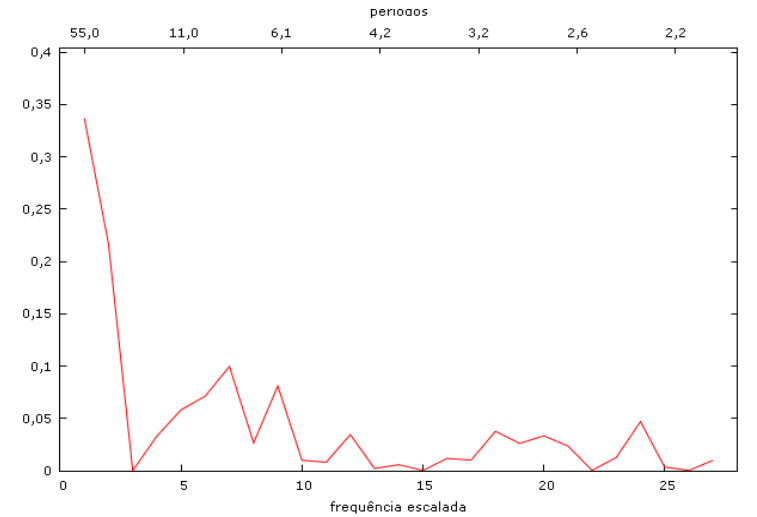

(b)

Figura 2 - Série de dados de expedição com transformação logarítmica (2a) e Periodograma (2b) apontando um comportamento não sazonal dos dados

Fonte: Autores (2018).

A escolha das ordens $(p, q)$ são fatores cruciais para o modelo ARMA de previsão (BUENO, 2008; STOCK; WATSON, 2012). Uma das formas de se fazer essa escolha é por meio das funções FAC e FACP (Figura 3).
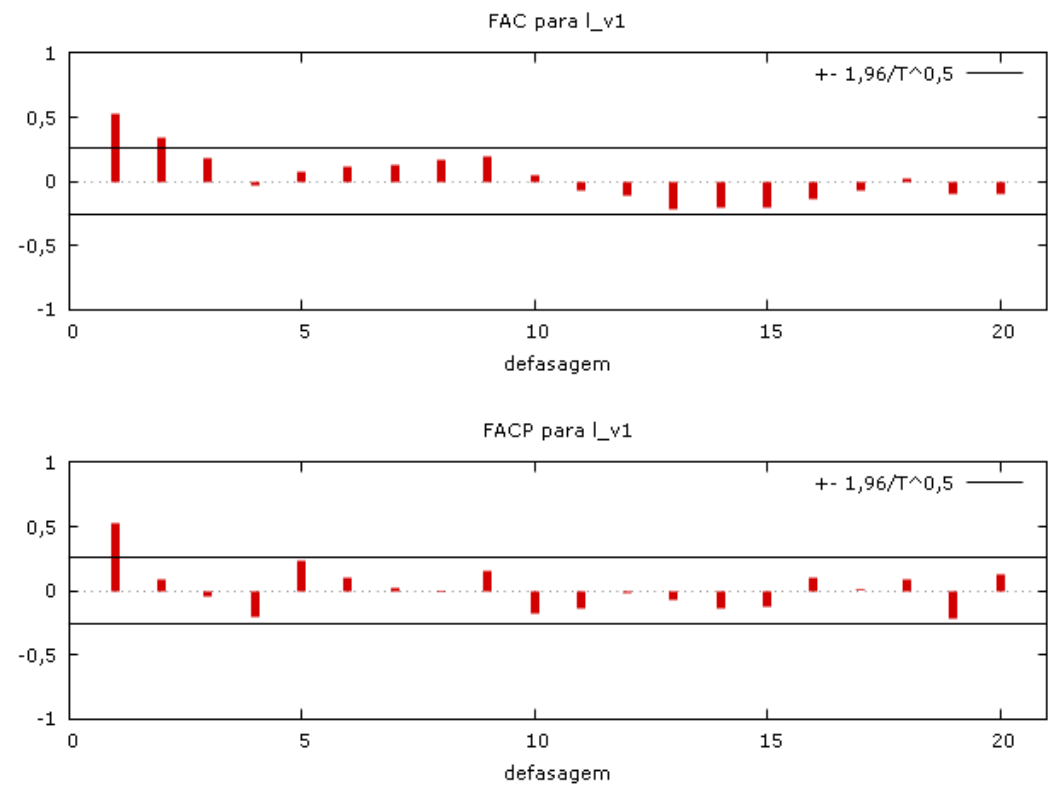

Figura 3 - FAC e FACP da série transformada Fonte: Autores (2018).

As primeiras defasagens da FAC evidenciam um possível modelo MA (2). A FACP é truncada a partir da primeira defasagem, o que revela a possibilidade de um modelo AR (1). 
Ao analisar em conjunto os dois correlogramas é possível considerar, também, o modelo $\operatorname{ARMA}(1,2)$.

$\mathrm{Na}$ Tabela 2 tem-se as combinações possíveis de modelo elaborados pela FAC e FACP. O Critério de Informação Bayesiano (BIC) e o Critério de Informação de Akaike (AIC), nas próximas colunas respectivamente, são os parâmetros de avaliação. Na última coluna é apresentado o valor $p$ do teste de Ljung-Box em que foi possível concluir, ao nível de 0,01 de significância, de que a hipótese nula dos resíduos serem ruído branco não deve ser rejeitada.

Tabela 2 - Avaliações dos modelos seguindo os critérios de BIC e AIC e o reste de Ljung-Box

\begin{tabular}{lccc}
\hline \multicolumn{1}{c}{ Modelo } & BIC & AIC & Ljung-Box \\
\hline ARMA (1,2) & 84,3248 & 74,2882 & 0,6646 \\
AR (1) & 77,6430 & 71,6210 & 0,7267 \\
MA (2) & 83,9295 & 75,9002 & 0,4166 \\
\hline
\end{tabular}

Fonte: Autores (2018).

Dentre os modelos testados, o modelo AR (1) foi eleito como o melhor, visto que o mesmo apresentou os menores valores de AIC e BIC. Contudo, além de verificar se os resíduos não são autocorrelacionados, tem-se a necessidade de verificar a pressuposição de normalidade.

Para a análise dos resíduos foi elaborado o teste para a hipótese nula de distribuição normal, no qual a mesma não foi rejeitada ao nível de 0,01 (valor $p=0,1383$ ). As Figuras das funções de autocorrelações destes resíduos são apresentados na Figura 4, em que é possível identificar que os resíduos não são autocorrelacionados.
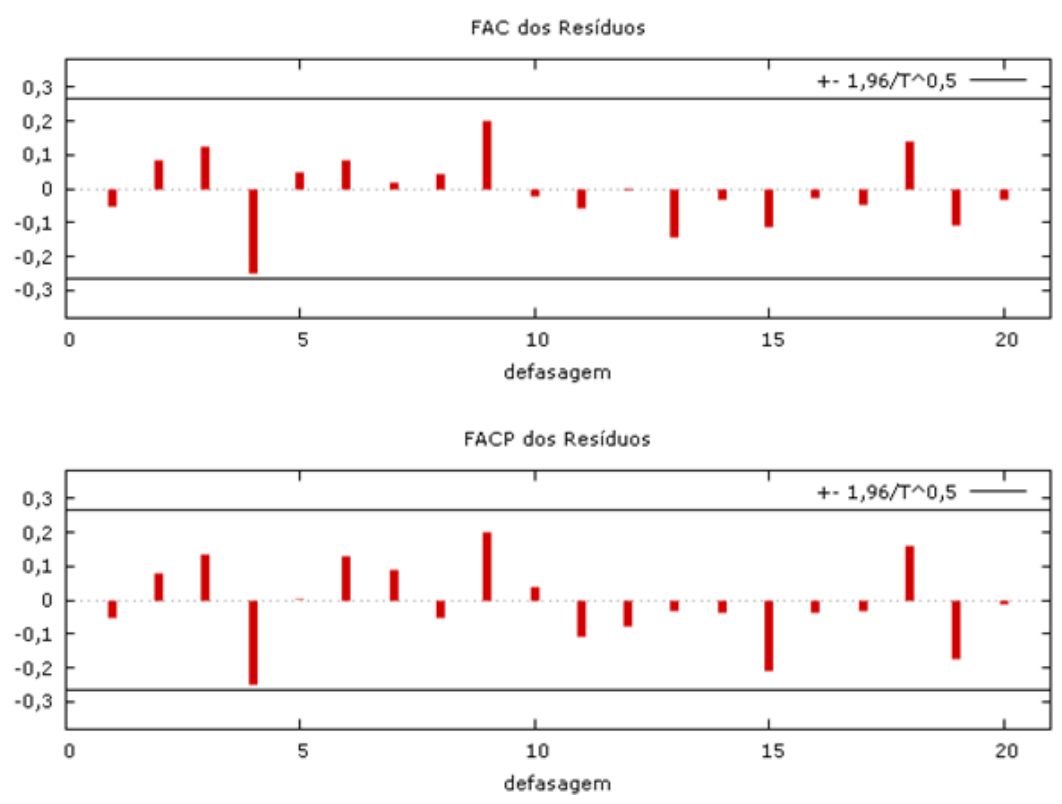

Figura 4 - FAC e FACP dos resíduos

Fonte: Autores (2018). 
A Tabela 3 foi elaborada para permitir a visualização dos valores reais e dos preditos com o modelo ajustado, AR (1), na grandeza real das expedições. Foi calculado o RMSE, cuja medida é utilizada para avaliação da previsão.

Tabela 3 - Valores Reais e preditos da expedição (x1000)

\begin{tabular}{lccccc}
\hline & Semana 56 & Semana 57 & Semana 58 & Semana 59 & RMSE \\
\hline Real & 447 & 754 & 583 & 292 & 222,96 \\
Predito & 465 & 381 & 341 & 321 & \\
\hline \multicolumn{5}{c}{ Fonte: Autores (2018). }
\end{tabular}

A seguir, na Figura 5, é possível visualizar toda a série com a aplicação do modelo AR (1). É possível perceber, por meio da figura, que os valores observados se encontram dentro do intervalo de confiança de $95 \%$, evidenciando um desempenho adequado do modelo.

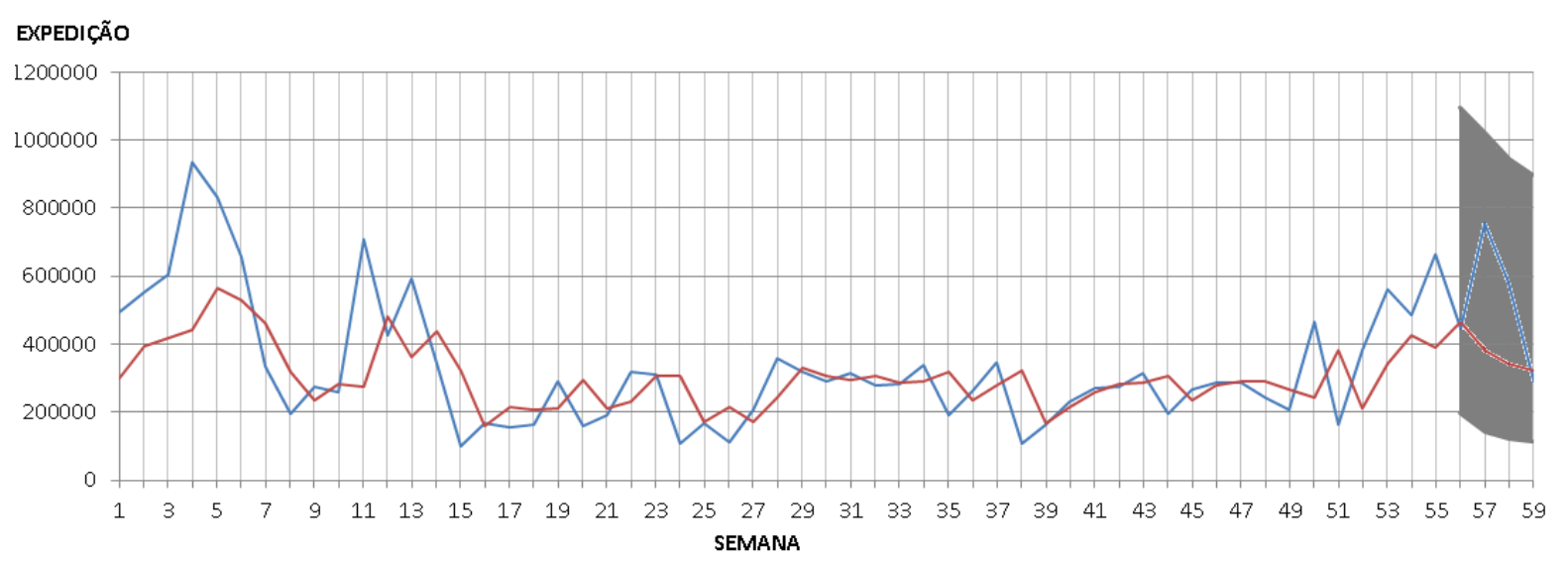

Figura 5 - Valores reais (linha azul), preditos (linha vermelha) e as bandas de 95\% de confiança (preenchimento em cinza) fornecidos pelo modelo proposto para as semanas 56 a 59

Fonte: Autores (2018).

Conforme visto, o resultado do RMSE apresentou um valor elevado, representando uma diferença de 42,96\% em relação ao valor médio observado (519). Este valor foi influenciado pelo alto índice expedição na semana 57. Todavia, é possível perceber que para as demais semanas os valores preditos pelo modelo proposto ficaram próximos aos observados. Para trabalhos futuros, tendo uma justificativa para o alto índice na semana 57, pode-se utilizar uma análise de intervenção em série temporal, pois pode dar possibilidade de que o modelo seja capaz de capturar este comportamento atípico. A obtenção de dados futuros também pode auxiliar a avaliação do modelo e, consequentemente, melhorar a previsão. Por fim, outra discussão possível para trabalhos posteriores é que sejam consideradas variáveis exógenas ao modelo e que podem influenciar o comportamento das expedições e vendas, como a conjuntura econômica e projeções para o agronegócio. 


\section{CONCLUSÃO}

Este estudo objetivou propor um modelo autorregressivo para previsão de demanda de pintainhos de corte de uma grande empresa localizada no centro-oeste de Minas Gerais. Diferentes métodos foram testados e avaliados sobre a série histórica que continha dados reais de expedição a fim de buscar pelo melhor modelo. O modelo autorregressivo de primeira ordem AR (1) foi escolhido, dados os critérios BIC e AIC. O modelo eleito foi utilizado para testar dados de quatro semanas, alcançando resultados dentro do intervalo de confiança estipulado.

Sabe-se que fazer previsões, especialmente quando se utiliza dados reais, é uma tarefa complexa e morosa, visto que as empresas estão sujeitas a diversos fatores externos, variáveis exógenas e incertezas que dificilmente podem ser antevistas. As previsões por si só não podem ser parâmetro de decisão de uma produção, pois é necessário levar em consideração outros aspectos intrínsecos ao ambiente empresarial. Para isso, sugere-se que nas análises estatísticas de previsão, utilizando ferramentas de séries temporais, sejam consideradas intervenções e aspectos que influenciam o comportamento da variável que está em investigação. Além disso, ela pode ser utilizada como ferramenta de apoio à tomada de decisão. Com isso as empresas podem ajustar o planejamento estratégico de longo prazo de forma mais assertiva.

A aceitabilidade do modelo foi evidenciada pela adequação do AR (1) à série de expedição investigada, respondendo ao objetivo principal do estudo. Os testes realizados demonstraram que a série não possuía tendência e nem sazonalidade. $O$ modelo eleito atendeu às necessidades da empresa, uma vez que ele foi capaz de fazer as previsões reais dos dados dentro do intervalo de confiança. Mais dados são necessários para fazer melhores ajustes no modelo. Além disso, é possível testar modelos probabilísticos, bem como verificar uma possível sazonalidade em períodos mais longos (longterm) ou muito curtos (very short term).

Tão importante quanto apresentar o modelo foi cumprir, por meio das avaliações estatísticas, as adequações do modelo à série investigada. Verificou-se, por exemplo, a inexistência do comportamento sazonal, bem como tendência de crescimento e decrescimento das demandas pelo produto ao longo de um ano. Por fim, este estudo buscou contribuir no acervo de estudos sobre previsões de séries temporais no que tange o ramo da avicultura e agroindústria, utilizando dados reais de expedição de pintos da linhagem de corte. 


\section{REFERÊNCIAS}

ABPA. Associação brasileira de proteína animal. Relatório anual ABPA 2019. 167p, 2019. Disponível em http://abpa-br.com.br/setores/avicultura/publicacoes/relatorios-anuais. Acessado em 28 set. 2019.

ALVAREZ-RAMIREZ, J.; RODRIGUEZ, E. AR (p)-based detrended fluctuation analysis. Physica A: Statistical Mechanics and its Applications, v. 502, p. 49-57, 2018.

ALVES, M. A. et al. An extension of nonstationary fuzzy sets to heteroskedastic fuzzy time series. In: EUROPEAN SYMPOSIUM ON ARTIFICIAL NEURAL NETWORKS, COMPUTATIONAL INTELLIGENCE AND MACHINE LEARNING (ESANN), 26th., Bruges. 2018. Proceedings [...]. Bruges, v. 1, p. 591-596, 2018.

BOX, G. E. P. et al. Time series analysis: forecasting and control. John Wiley \& Sons, 2015.

BRANCO, A, N. et al. Dimensionamento da oferta de produtos perecíveis a uma rede varejista por meio de métodos de previsão. Revista Gestão Industrial, Ponta Grossa, v. 14, n. 4, p. 240-258, 2018.

BUENO, R. L. S. Econometria de séries temporais. São Paulo: Cengage Learning, 2008, $300 \mathrm{p}$.

CHIA VENATO, I. Planejamento e controle da produção. 2. ed. Barueri: Manole, 2008, $138 \mathrm{p}$.

DELABOUGLISE, A.; et al. Poultry population dynamics and mortality risks in smallholder farms of the Mekong river delta region. BMC Veterinary Research, London, v. 15, n. 1, p. $205,2019$.

DE MEDEIROS, E. S. et al. Modelo SARIMAX aplicado aos casos de notificações de dengue em Recife, PE. Matemática e Estatística em Foco, Uberlândia, v. 6, n. 1, p. 01-11, 2018.

GRETL - GNU Regression, Econometricsand Time-Series Library. 2018. Disponível em http://gretl.sourceforge.net/pt.html. Acesso em: 17 jul. 18.

KRUSKAL, W. H.; WALLIS, W. A.. Use of ranks in one-criterion variance analysis. Journal of the American Statistical Association, Boston, v. 47, n. 260, p. 583-621, 1952.

LEMOS, F. O. Metodologia para seleção de métodos de previsão de demanda. 2016. 183 f. Dissertação (Mestrado em Engenharia Elétrica) - Universidade Federal do Rio Grande do Sul, Porto Alegre, 2016.

MARTINS, P. G.; LAUGENI, F. P. Administração da produção. 2. ed. São Paulo: Saraiva, 2005. 562 p.

MORETTIN, P. A.; TOLOI, C. M. C. Análise de séries temporais: modelos lineares univariados. 3. ed., São Paulo: Blucher, 2018. 474 p. 
OLIVEIRA, E. S.; DOURADO, J. D. A.; MELLO, J. A. V. B. Aplicação de modelos de previsão de demanda em uma fábrica de embalagens plásticas. Revista da Universidade Vale do Rio Verde, Três Corações, v. 15, n. 2, p. 354-373, 2017.

OSINOWO, O. H.; TOLORUNJU, E. T. Technical efficiency of poultry egg production in Ogun state, Nigeria. Journal of Agribusiness and Rural Development, Poznań, v. 51, n. 1, p. 51-58, 2019.

PAIVA, D. A.; HERVAL, A. C. F.; SÁFADI, T.. Metodologia de séries temporais como ferramenta de análise na produção de frangos no Brasil. Sigmae, Alfenas, v. 8, n. 2, p. 227-237, 2019.

PAPARODITIS, E.; POLITIS, D. N. The asymptotic size and power of the Augmented Dickey-Fuller test for a unit root. Econometric Reviews, v. 37, n. 9, p. 955-973, 2018.

RODRIGUES, W. et al. Competitividade do sistema agroindustrial do frango de corte no Tocantins: o caso da empresa Frango Norte. REGE-Revista de Gestão, São Paulo, v. 18, n. 2, p. 195-209, 2011.

SANTOS, Á. A. et al. Método SARIMA aplicado para previsão de demanda em uma loja varejista de alimentos naturais. In: SIMPÓSIO DE INOVAÇÃO, EMPREENDEDORISMO E SETOR PÚBLICO - SIEGEP, III., Lavras, 2019. Anais [...]. Lavras, MG, 2019. p. 122-132.

SCHMIDT, N. S.; SILVA, C. L. Pesquisa e desenvolvimento na cadeia produtiva de frangos de corte no Brasil. Revista de Economia e Sociologia Rural, Brasília, v. 56, n. 3, p. 467-482, 2018.

SILVA, P. C. D. L. et al. Probabilistic Forecasting with Seasonal Ensemble Fuzzy Time-Series. In: CONGRESSO BRASILEIRO DE INTELIGÊNCIA COMPUTACIONAL, XIII., 2017, Rio de Janeiro/RJ. Anais [...] Rio de Janeiro, 2017.

SIMCHI-LEVI, D.; KAMINSKY, P.; SIMCHI-LEVI, E. Cadeia de suprimentos: projeto e gestão. 3. ed. Porto Alegre: Bookman, 2010. 586 p.

STOCK, J. H.; WATSON, M. W. Introduction to econometrics: global edition. Boston: Pearson Education, 2012.

TANAKA, K.. Time series analysis: nonstationary and noninvertible distribution theory. John Wiley \& Sons, 2017.

THEOBALD, S. et al. Antimicrobial resistance trends in Escherichia coli in South African Poultry: 2009-2015. Foodborne pathogens and disease, New Rochelle, v. 16, n. 9, 2019.

TUBINO, D. F. Planejamento e controle da produção: teoria e prática. 3. ed. São Paulo: Atlas, 2017, 304 p.

UEDA, R. M. et al. Combination of demand forecasting techniques: application in an animal nutrition agroindustry. Exacta - EP, São Paulo, v. 16, n. 2, p. 83-93, 2018. 


\section{DADOS DOS AUTORES}

\section{Álvaro de Aquino Santos}

E-mail: aasalvarosamonte@gmail.com

Currículo lattes: http://lattes.cnpq.br/1456734236848533

Graduando em Administração pelo Instituto Federal de Educação, Ciência e Tecnologia de Minas Gerais, Campus Formiga - MG.

\section{Marcos Antônio Alves}

E-mail: m.voicer@gmail.com

Currículo lattes: $\underline{\text { http://lattes.cnpq.br/4636940519958225 }}$

Doutorando (2018--) e Mestre (2016-2018) em Engenharia Elétrica pela Universidade Federal de Minas Gerais (UFMG) na linha de pesquisa de Inteligência Computacional. Possui interesse em Ciência de Dados, Aprendizado de Máquina, Tomada de Decisão Multicritério, Computação Evolucionária, Lógica Fuzzy e Sistemas de Recomendação.

\section{Elias Silva de Medeiros}

E-mail: eliasestatistica@gmail.com

Currículo lattes: http://lattes.cnpq.br/5052910988468793

Possui graduação em Estatística pela Universidade Estadual da Paraíba (2011), mestrado em Estatística e Experimentação Agronômica pela Escola Superior de Agricultura 'Luiz de Queiroz' - USP (2014) e doutorado em Estatística e Experimentação Agropecuária pela Universidade Federal de Lavras (2018). Atualmente é professor adjunto da Universidade Federal da Grande Dourados. Possui interesse na área de Probabilidade e Estatística com foco em análise de séries temporais.

\section{Igor Neves Nunes}

E-mail: igor--neves@hotmail.com

Graduando em Administração pelo Instituto Federal de Educação, Ciência e Tecnologia de Minas Gerais, Campus Formiga - MG.

\section{Rafael João de Melo Miguel Cardoso}

E-mail: rafael.joao.melo@ gmail.com

Graduando em Administração pelo Instituto Federal de Educação, Ciência e Tecnologia de Minas Gerais, Campus Formiga - MG. 